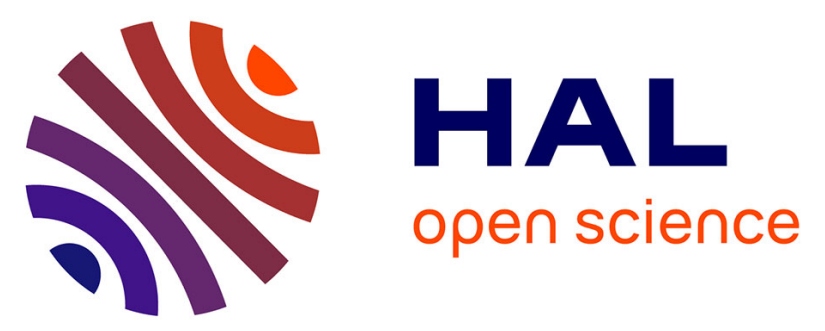

\title{
Note sur une anomalie du spectre de phonons associée au contact d'une bande d'énergie avec la surface de fermi ; conséquence pour la stabilité du métal
}

\author{
L. Dagens
}

\section{- To cite this version:}

L. Dagens. Note sur une anomalie du spectre de phonons associée au contact d'une bande d'énergie avec la surface de fermi; conséquence pour la stabilité du métal. Journal de Physique Lettres, 1976, 37 (2), pp.37-40. 10.1051/jphyslet:0197600370203700 . jpa-00231229

HAL Id: jpa-00231229

https://hal.science/jpa-00231229

Submitted on 1 Jan 1976

HAL is a multi-disciplinary open access archive for the deposit and dissemination of scientific research documents, whether they are published or not. The documents may come from teaching and research institutions in France or abroad, or from public or private research centers.
L'archive ouverte pluridisciplinaire HAL, est destinée au dépôt et à la diffusion de documents scientifiques de niveau recherche, publiés ou non, émanant des établissements d'enseignement et de recherche français ou étrangers, des laboratoires publics ou privés. 


\title{
NOTE SUR UNE ANOMALIE DU SPECTRE DE PHONONS ASSOCIÉE AU CONTACT D'UNE BANDE D'ÉNERGIE AVEC LA SURFACE DE FERMI; CONSÉQUENCE POUR LA STABILITÉ DU MÉTAL
}

\author{
L. DAGENS \\ Commissariat à l'Energie Atomique, Centre d'Etudes de Limeil, \\ B.P. 27, 94190 Villeneuve-Saint-Georges, France
}

(Reçu le 21 novembre 1975, révisé le 3 décembre 1975, accepté le 4 décembre 1975)

\begin{abstract}
Résumé. - La polarisabilité à $T=0 \mathrm{~K}$ d'un métal est une fonction non analytique de volume $V$ en chaque point $V=V_{\mathrm{c}}$ où l'écart $\Delta$ entre une bande d'énergie et le niveau de Fermi s'annule. On établit la forme de l'anomalie (pour $\Delta>0$ ) du spectre de phonons et de l'énergie libre $E_{\mathrm{ph}}: E_{\mathrm{ph}}$ comporte un terme en $-C\left(V-V_{c}\right)^{3 / 2}$ avec $C>0$, ce qui implique l'existence d'une instabilité isostructurale pour $T$ suffisamment petit.
\end{abstract}

\begin{abstract}
The $T=0 \mathrm{~K}$ density response function in a metal is a non-analytic function of the volume $V$ at any $V=V_{\mathrm{c}}$ such that the gap $\Delta$ between an excited band and the Fermi level vanishes. Explicit forms of the corresponding anomalies of the phonon spectrum and free energy $E_{\mathrm{ph}}$ are derived (for $\Delta>0$ ) in the framework of the adiabatic and quasi-harmonic approximation : $E_{\mathrm{ph}}$ involves a $-C\left(V-V_{\mathrm{c}}\right)^{3 / 2}$ anomaly with $C>0$, when $T=0 \mathrm{~K}$. It is inferred that the metal is unstable with respect to an isostructural phase transition when $T$ is small enough.
\end{abstract}

1. Introduction et formulation générale. - On considère un métal dont une bande d'énergie $E_{\mathrm{d}}(\mathbf{k})$ (minimum au point de symétrie $\mathbf{k}_{\mathrm{d}}$ ) entre en contact avec la surface de Fermi $\left(S_{\mathrm{F}}\right)$ sous l'effet d'une compression. La topologie de $S_{\mathrm{F}}$ est alors modifiée et ceci est à l'origine d'anomalies électroniques qui ont été décrites et étudiées par Lifshitz [1]. On suppose que l'écart

$$
\Delta(V)=E_{\mathrm{d}}\left(\mathbf{k}_{\mathrm{d}}\right)-E_{\mathrm{F}}
$$

entre la bande et $E_{\mathrm{F}}$ s'annule pour $V=V_{\mathrm{c}}$ et que sa dérivée par rapport à $V, \Delta^{\prime}\left(V_{\mathrm{c}}\right)$, est strictement positive. Une petite poche d'états occupés apparaît pour $V<V_{\text {c }}$. Il lui correspond une anomalie de la pression électronique, négative, en $\left(V_{c}-V\right)^{3 / 2}$ susceptible d'induire, sous certaines conditions, une transition isostructurale du métal [1]. On sait également que lorsque le niveau $E_{\mathrm{d}}\left(\mathbf{k}_{\mathrm{d}}\right)$ qui touche $S_{\mathrm{F}}$ est dégénéré, il peut $\mathrm{y}$ avoir aussi une instabilité vis-à-vis d'une déformation du cristal [2], associée à une anomalie des constantes élastiques lorsque $V=V_{c}$.

On étudie ici un problème similaire concernant le spectre de phonons d'un métal et les fonctions thermodynamiques associées, lorsque la nouvelle poche est sur le point de se former $\left(V>V_{\mathrm{c}}\right)$. L'écart $\Delta(V)$ tend alors vers zéro : la polarisabilité des électrons de conduction s'accroît lorsque $\Delta \rightarrow 0(\Delta>0)$ et présente une anomalie lorsque $V=V_{\mathrm{c}}$. Ceci implique l'apparition d'anomalies nouvelles du spectre de phonons; en fait, il apparait une surface entière d'anomalies de Kohn [3] lorsque $V=V_{c}$, pour tout q joignant un point de $S_{\mathrm{F}}$ à $\mathbf{k}_{\mathrm{d}}$ (où la surface de Fermi se réduit à un point quand $V=V_{\mathrm{c}}$ ). Il en résulte que l'énergie libre $E_{\mathrm{ph}}$ des phonons (par exemple) présente une anomalie en $V=V_{\mathrm{c}}$; on établit ici que, dans la limite des approximations énoncées plus loin, cette anomalie est suffisamment forte (en $\left.\left(V-V_{\mathrm{c}}\right)^{3 / 2}, V>V_{\mathrm{c}}\right)$ pour que le métal soit instable vis-à-vis d'une compression isostructurale, pour un volume $V$ légèrement supérieur à $V_{\mathrm{c}}$.

Le contexte physique est le suivant. On sait que le césium présente une transition isostructurale $(\mathrm{cfc} \rightarrow \mathrm{cfc})$ à 42,2 kbars [4] $\left(V / V_{0}=0,45\right)$. La structure de bande du Cs comprimé a été calculée par Yamashita et Asano [5] (voir aussi Louie et Cohen [6]). Ils trouvent que le niveau $\mathrm{X}_{3}$ (en $\left.\mathbf{k}_{\mathrm{d}}=(0,0,1) 2 \pi / a\right)$ est égal à $E_{\mathrm{F}}$ pour $V_{\mathrm{c}} \approx 0,47 V_{0}$. Ceci est assez voisin du volume de la transition CsII $\rightarrow$ CsIII à 42,2 kbars. Ce fait a été noté et utilisé par plusieurs auteurs $[5,6,7,8]$ sans que la discontinuité du volume ait été expliquée. Peut-être sera-t-il possible de l'expliquer comme une conséquence de l'anomalie de l'interaction électron-phonon due au contact des bandes d (aux trois points $\mathrm{X}_{3}$ ) avec $S_{\mathrm{F}}$. 
On s'est limité aux approximations suivantes. Les fonctions thermodynamiques sont évaluées à l'approximation quasi harmonique (non self-consistante). Le comportement du spectre de phonons est étudié à l'approximation adiabatique, en utilisant la formulation de Pick, Cohen et Martin [9]; la matrice diélectrique $\varepsilon\left(\mathbf{Q}^{\prime}, \mathbf{Q}\right)$ qui y intervient est calculée à $T=0 \mathrm{~K}$ (on néglige l'effet de l'agitation thermique des électrons et de celle du réseau sur la fonction réponse en densité des électrons).

On procède de la façon suivante. La matrice diélectrique s'écrit $1-v_{\mathrm{cb}} \pi$ (éq. (5.1) de [9]) où $\pi$ est la polarisabilité irréductible exacte du métal et $v_{\mathrm{cb}}$ l'interaction coulombienne. $\pi$ est écrit, d'après Hubbard [9], sous la forme $\chi /\left(1-v_{\text {xc }} \chi\right)$ où $\chi$ est la polarisabilité irréductible R.P.A. (éq. (7), plus loin) et où l'interaction d'échange et corrélation $v_{x c}$ est précisément définie par cette relation. La fonction réponse $\chi$ est séparée en $\chi_{0}+\delta \chi$, où $\delta \chi$ inclut tout ce qui est singulier à la limite $\Delta \rightarrow 0\left(V \rightarrow V_{\mathrm{c}}\right)$. On calcule la variation $\delta \omega_{q}(q=\mathbf{q}, \lambda)$ du spectre de phonons au premier ordre en $\delta \chi$. Elle est donnée par les formules de perturbation suivantes. La matrice dynamique $\operatorname{MD}(q)$ s'écrit $C(q)-C(0)$ (éq. (4.4) de [9]) pour un réseau de Bravais et $C(q)$ s'exprime en fonction de $\varepsilon^{-1}$ (éq. (4.5) de [9]). La contribution $\delta C$ associée à $\delta \chi$ se calcule aisément et s'écrit

$$
\delta C_{\alpha \beta}(q)=\Omega_{\mathbf{a}} \sum_{\mathbf{g g}^{\prime}} F_{\alpha}^{*}(\mathbf{Q}) F_{\beta}\left(\mathbf{Q}^{\prime}\right) \delta \chi\left(\mathbf{Q}, \mathbf{Q}^{\prime}\right)
$$

avec les notations suivantes. Les sommations portent sur les vecteurs $\mathbf{g}, \mathbf{g}^{\prime}$ du réseau réciproque $; \mathbf{Q}=\mathbf{g}+\mathbf{q}$, etc.; $\Omega_{\mathrm{a}}$ est le volume atomique. Le vecteur $\mathbf{F}$, enfin, est défini par

$$
F_{\alpha}(\mathbf{Q})=\frac{Z_{\mathrm{N}}}{\Omega_{\mathrm{a}}} \sum_{\mathbf{g}^{\prime \prime}} \widetilde{\varepsilon}^{-1}\left(\mathbf{Q}, \mathbf{Q}^{\prime \prime}\right) v_{\mathrm{cb}}\left(Q^{\prime \prime}\right) i Q_{\alpha}^{\prime \prime}
$$

où $Z_{\mathrm{N}}$ est la charge nucléaire et $\widetilde{\varepsilon}^{-1}$ la matrice inverse de $1-\left[v_{\mathrm{cb}}+v_{\mathrm{xc}}\right] \chi$. Notons qu'une approximation dú pseudo-potentiel (local) donnerait

$$
\mathbf{F}=i \mathbf{Q} V(Q)
$$

(avec $V(Q)=v_{\text {ion }}(Q) / \Omega_{\mathrm{a}} \widetilde{\varepsilon}$, coefficient de Fourier du pseudo-potentiel self-consistant).

Dans le cas présent, $\delta \chi$ est nul pour $\mathbf{q}=0$ et $\delta D$ est simplement égal à $\delta C(q) / M$. La variation $\delta \omega_{q}$ de $\omega_{q}$ est donnée par la formule de perturbation bien connue [10], $\mathbf{e}_{q} \cdot \delta D \cdot \mathbf{e}_{q}\left(\mathbf{e}_{q}\right.$ est la polarisation du phonon $q=\mathbf{q}, \lambda$ ), d'où la formule

$2 \omega_{q} \delta \omega_{q}=\frac{\Omega_{\mathrm{a}}}{M} \sum_{\mathbf{g g}^{\prime}} \mathbf{e}_{q} \cdot \mathbf{F}(\mathbf{Q}) \delta \chi\left(\mathbf{Q}, \mathbf{Q}^{\prime}\right) \mathbf{e}_{q} \cdot \mathbf{F}^{*}\left(\mathbf{Q}^{\prime}\right)$

qui sera utilisée plus loin pour le calcul de $\delta E_{\mathrm{ph}}$ (partie non analytique de l'énergie libre des phonons). On note le caractère symétrique de $(5)$, d'où il résulte que $\delta \omega_{q}$ a un signe bien défini, celui de la matrice hermitienne $\delta \chi\left(Q, Q^{\prime}\right)$, si cette matrice est non positive ou non négative.
Soit $f(\omega)$ une fonction quelconque de $\omega$. On désigne par $\left\langle f\left(\omega_{q}\right)\right\rangle$ la moyenne de $f$ sur le spectre de phonon et par $\delta\left\langle f\left(\omega_{q}\right)\right\rangle$ la variation de cette moyenne quand $\chi_{0}$ est accrue de $\delta \chi$, à $0\left(\delta \chi^{2}\right)$ près. Son expression est

$$
\delta\left\langle f\left(\omega_{q}\right)\right\rangle=\frac{1}{V_{\mathrm{ZB}}} \int_{\mathrm{ZB}} \mathrm{dq}\left\langle\delta \omega_{q} \frac{\partial f}{\partial \omega_{q}}\right\rangle_{\text {pol }}
$$

où $\delta \omega_{q}$ doit être remplacée par son expression. (5). La moyenne $\langle\cdots\rangle_{\text {pol }}$ porte sur les trois polarisations $\lambda=1,2$ et 3 d'un phonon $q=\mathbf{q}, \lambda$. L'intégration en dq est sur la première zone de Brillouin (ZB) et $V_{\mathrm{ZB}}$ en est le volume. Cette formule est utilisée section 3 pour le calcul de la partie singulière de l'énergie libre.

2. Expression générale de $\delta \chi$. - On étudie ici le comportement de $\chi$ à la limite $\Delta \rightarrow 0(\Delta>0)$. Une expression générale de $\chi$ est

$$
\begin{aligned}
\chi\left(\mathbf{Q}^{\prime} \mathbf{Q}\right) & =-\sum_{\mathbf{b}} \frac{4}{(2 \pi)^{3}} \times \\
& \times \int \mathrm{d} \mathbf{k} J_{s \mathbf{b}}\left(\mathbf{Q}^{\prime}, \mathbf{Q}, \mathbf{k}\right) \frac{f_{s}(\mathbf{k})}{E_{\mathbf{b}}(\mathbf{k}+\mathbf{q})-E_{s}(\mathbf{k})}
\end{aligned}
$$

$s$ désigne la bande contenant les états occupés. $\mathbf{Q}=\mathbf{q}+\mathbf{g}$, etc., avec $\mathbf{q}$ dans la première zone de Brillouin. La sommation porte sur toutes les bandes b. $J_{s \mathrm{~b}}$ est un produit d'éléments de matrice du type

$$
\langle\mathbf{b}, \mathbf{k}+\mathbf{q}|\exp i \mathbf{Q} \cdot \mathbf{r}| s, \mathbf{k}\rangle .
$$

On ne s'intéresse qu'à cette fraction $\delta \chi$ qui devient singulière pour $\Delta=0$. Seule la bande $b=d$ contribue. De plus, l'intégration en $\mathbf{k}$ peut être limitée à cette petite région pour laquelle l'énergie d'excitation $E_{\mathrm{d}}-E_{\mathrm{s}}$ reste petite et peut s'annuler quand $\Delta=0$. Ces états $\mathbf{k}$ doivent vérifier les propriétés suivantes : (a) $\mathbf{k}$ doit être voisin de $S_{\mathbf{F}}$; (b) $\left\{\mathbf{k}+\mathbf{q}-\mathbf{k}_{\mathrm{d}}\right\}$ doit être voisin de $0(\{\cdots\}$ dénote le vecteur réduit dans la première zone de Brillouin). Rappelons que $E_{\mathrm{d}}\left(\mathbf{k}_{\mathrm{d}}\right)$ est le minimum de $E_{\mathrm{d}}(\mathbf{k})\left(\mathbf{k}_{\mathrm{d}}=(001) 2 \pi / a\right.$ pour le Cs cfc). Il en résulte que $\left\{\mathbf{k}_{\mathrm{d}}-\mathbf{q}\right\}$ doit être voisin de $S_{\mathrm{F}}$. Il est alors commode de caractériser $\mathbf{q}$ par les vecteurs $K_{F}(q)$ et $\tau(q)$ où $K_{F}$ repère le pied de la plus courte normale abaissée de $\left\{\mathbf{k}_{\mathrm{d}}-\mathbf{q}\right\}$ sur $S_{\mathrm{F}}$ et $\tau$ joint $\mathbf{K}_{\mathbf{F}}$ à $\left\{\mathbf{k}_{\mathbf{d}}-\mathbf{q}\right\}$

$$
\mathbf{q}=\left\{\mathbf{k}_{\mathbf{d}}-\left(\mathbf{K}_{\mathbf{F}}+\tau\right)\right\} ;
$$

$|\tau|$ est la distance entre $\left\{\mathbf{k}_{\mathrm{d}}-\mathbf{q}\right\}$ et $S_{\mathrm{F}} . \delta \chi$ peut être pris égal à zéro là où $|\tau|$ reste supérieur à un nombre positif $\tau_{0}$ arbitrairement petit mais indépendant de $\Delta$.

D'après ce qui est dit au paragraphe précédent, on peut limiter l'intégration en $\mathbf{k}$ à un domaine arbitrairement petit, centré sur $\mathbf{K}_{\mathrm{F}}$. On adopte un système local de coordonnées curvilignes $\mathbf{s}(\mathbf{k})$ tel que (a) $E_{s}(\mathbf{k})=E_{\mathrm{F}}+\mathbf{s} . \mathbf{v}_{\mathrm{F}}$ pour $\quad|\mathbf{s}| \gtrless s_{0} \ll k_{\mathrm{FO}}$; 
$\mathbf{s}\left[\mathbf{K}_{\mathbf{F}}(\mathbf{q})\right]=0$; et (c) le jacobien $\partial(\mathbf{k}) / \partial(\mathbf{s})$ est égal à 1 en $\mathbf{s}=0$. Ceci implique

$$
\mathbf{v}_{\mathrm{F}}=\operatorname{grad}_{\mathbf{k}} E_{\mathrm{s}}\left(\mathbf{K}_{\mathrm{F}}\right) \text {. }
$$

On développe l'énergie d'excitation $E_{\mathrm{d}}-E_{s}$ sous la forme

$$
\begin{aligned}
E_{\mathrm{d}}\{\mathbf{k}+\mathbf{q}\}-E_{s}\{\mathbf{k}\} & =\Delta+ \\
& +\frac{\hbar^{2}}{2 m_{\mathrm{d}}}\left(\tau^{2}+s^{2}\right)-\mathbf{v}_{\mathbf{F}} \cdot \mathbf{s}+\mathrm{O}_{3} .
\end{aligned}
$$

On a négligé le caractère anisotrope de la bande $\mathrm{d}$ $\left(m_{\mathrm{d}}\right.$ est une masse effective moyenne). $\mathbf{v}_{\mathrm{F}}^{\prime}$ est la différence des vitesses $\mathbf{v}_{\mathrm{F}}-\hbar \tau / m_{\mathrm{d}}$ égale à $\mathbf{v}_{\mathrm{F}}$ à $0\left(\tau_{0}\right)$ près.

On obtient ainsi l'expression suivante de $\delta \chi$,

$$
\begin{aligned}
\delta \chi=-\frac{4 J_{s d}\left(\mathbf{Q}^{\prime}, \mathbf{Q}, \mathbf{K}_{\mathbf{F}}\right)}{(2 \pi)^{3}} 2 \pi \int_{0}^{s_{0}} s^{2} \mathrm{~d} s \times \\
\quad \times \int_{0^{*}}^{1} \mathrm{~d} t \frac{2 m_{\mathbf{d}}}{2 m_{\mathrm{d}} \Delta+\hbar^{2}\left(\tau^{2}+s^{2}\right)+v_{\mathbf{F}} \hbar s t}
\end{aligned}
$$

exacte à $0\left(s_{0}\right)$ et $0\left(\tau_{0}\right)$ près. On a choisi $\mathbf{v}_{\mathbf{F}}$ comme axe d'intégration $\mathrm{O} z$. L'intégration en $t$ s'effectue immédiatement, et on trouve que seul le terme en

$$
\frac{1}{s} \log \left(2 m_{\mathrm{d}} \Delta+\hbar^{2}\left(\tau^{2}+s^{2}\right)\right)
$$

donne une contribution finale singulière pour $\Delta=|\tau|=0$ et doit être retenu. L'intégration en $s$ s'effectue alors exactement et donne, en introduisant la quantité sans dimension

$$
a=\left[m_{\mathrm{d}} \Delta+\frac{\hbar^{2}}{2}|\tau(\mathbf{q})|^{2}\right] /\left(\hbar k_{\mathrm{FO}} v_{\mathrm{F}}\right),
$$

$\delta \chi\left(\mathbf{Q}^{\prime}, \mathbf{Q}\right)=-J_{\mathrm{sd}}\left(\mathbf{Q}^{\prime}, \mathbf{Q}, \mathbf{K}_{\mathrm{F}}\right) \frac{m_{\mathrm{d}} k_{\mathrm{FO}}}{\pi^{2} \hbar^{2}} a \log a$.

$v_{\mathrm{F}}$ est donné par (9) et $k_{\mathrm{Fo}}$ est défini par $\left(3 \pi^{2} / \Omega_{\mathrm{a}}\right)^{1 / 3}$. Rappelons que $\mathbf{K}_{\mathbf{F}}$ et $\tau$ sont des fonctions de

$$
\mathbf{q}=\{\mathbf{Q}\}=\left\{\mathbf{Q}^{\prime}\right\}
$$

(voir (8)) et que l'on a posé $\delta \chi=0$ pour $\tau>\tau_{0}$.

3. L'énergie libre des phonons et conclusion. On examine d'abord le cas $T \ll \theta_{\mathbf{D}}$. L'énergie libre se réduit à l'énergie $E_{\mathrm{PZ}}$ (par atome) du point zéro. $E_{\mathrm{PZ}}$ est égale à trois fois la moyenne $\langle\hbar \omega / 2\rangle$ de $\hbar \omega / 2$, moyenne prise sur spectre de phonons. On calcule $\delta E_{\mathrm{PZ}}$ en substituant $(11)$ dans $(5,6)$ avec $f(\omega)$ remplacé par $3 \hbar \omega / 2$. Il s'agit d'extraire du résultat la partie singulière en $\Delta$. On a vu que $\delta \chi \neq 0$ pour $|\tau|<\tau_{0}$ seulement, avec $\tau_{0}$ arbitrairement petit. Exprimant $q$ en fonction de $\mathbf{K}_{\mathbf{F}}\left(\operatorname{sur} \boldsymbol{S}_{\mathrm{F}}\right)$ et de $\tau$ (éq. (8)), l'intégration en $\int d q$ peut s'écrire $\int d \sigma\left(\mathbf{K}_{F}\right) d \tau$ où $\mathrm{d} \sigma\left(\mathbf{K}_{\mathrm{F}}\right)$ est l'élément d'aire de $S_{\mathrm{F}}$. On effectue d'abord l'intégration en $\tau$ en négligeant toute variation en $\tau$ sauf celle de $a \log a$ (éq. (11)). Il apparait l'intégrale

$$
\begin{aligned}
\int_{-\tau_{0}}^{+\tau_{0}} \mathrm{~d} \tau a \log a= & 2 k_{\mathrm{FO}} v_{\mathrm{F}} \times \\
& \times \int_{-x_{0}}^{x_{0}} \mathrm{~d} x\left(a_{0}+x^{2}\right) \log \left(a_{0}+x^{2}\right)
\end{aligned}
$$

avec

et

$$
\begin{gathered}
a_{0}=m_{\mathrm{d}} \Delta /\left(v_{\mathrm{FO}} v_{\mathrm{F}}\right)\left(v_{\mathrm{FO}}=\hbar k_{\mathrm{FO}}\right) \\
\hbar \tau=\left(2 v_{\mathrm{FO}} v_{\mathrm{F}}\right)^{1 / 2} x .
\end{gathered}
$$

On montre aisément (en intégrant par parties) que l'intégrale en $x$ vaut $\pi a_{0}^{3 / 2}$ à part un terme (négligé) analytique en $a_{0}$. Revenant à $\delta E_{\mathrm{PZ}}$, on obtient en fin de compte

$$
\delta E_{\mathrm{PZ}}=-\left(2 m_{\mathrm{d}} \Delta\right)^{3 / 2} \frac{3}{V_{\mathrm{BZ}}} \int_{S_{\mathrm{F}}} \mathrm{d} \sigma\left(\mathbf{K}_{\mathrm{F}}\right)\left\langle\frac{1}{2} A(q)\right\rangle_{\mathrm{pol}}
$$

où l'intégrale de surface porte sur $\mathbf{K}_{\mathbf{F}}$ avec

$$
\mathbf{q}=\left\{\mathbf{k}_{\mathrm{d}}-\mathbf{K}_{\mathbf{F}}\right\}
$$

et où la moyenne est prise sur les trois polarisations du phonon; on a posé

$$
\begin{aligned}
& A(\mathbf{q})=\frac{\Omega_{\mathrm{a}}}{4 \pi \hbar^{3} M \omega_{q}} \times \\
& \quad \times \sum_{\mathbf{g g}} \mathbf{e}_{q} \cdot \mathbf{F}(\mathbf{Q}) \mathbf{e}_{q} \cdot \mathbf{F}\left(\mathbf{Q}^{\prime}\right) J_{s d}\left(\mathbf{Q}, \mathbf{Q}^{\prime}, \mathbf{K}_{\mathbf{F}}\{\mathbf{q}\}\right) .
\end{aligned}
$$

On note que $J_{s d}\left(\mathbf{Q}, \mathbf{Q}^{\prime}, \mathbf{K}_{\mathrm{F}}\right)$ est une matrice hermitienne non négative. $A(q)$ est en conséquence positif : le signe de $\delta E_{\mathrm{PZ}}$ est connu sans ambiguïté.

Remarque. - Si le point de symétrie $\mathbf{k}_{\mathrm{d}}$ est dégénéré d'ordre $r, A(q)$ doit être multiplié par $r$ (soit $r=3$ pour le point $\mathrm{X}_{3}$ du Cs $\mathrm{cfc}$ ).

La même méthode permet d'évaluer $\delta\langle f(\omega)\rangle$ pour une fonction $f$ quelconque (voir la formule (6)).

On obtient (12) avec $\frac{\partial f}{\partial \omega_{q}} A(q)$ au lieu de $\frac{1}{2} A(q)$. On a donc établi que toute moyenne $\left\langle f\left(\omega_{q}\right)\right\rangle$ comporte une contribution singulière en $\Delta^{3 / 2}(\Delta>0)$. Appliquant ce résultat à l'énergie libre $E_{\mathrm{ph}}(V, T)$ (approximation quasi-harmonique), on voit que $\delta E_{\mathrm{ph}}$ se comporte en $\Delta^{3 / 2}$. Explicitant le résultat pour $T \gg \theta_{\mathrm{D}}$ on obtient, avec $\partial f / \partial \omega$ égal à $3 k_{\mathrm{B}} T / \hbar \omega$,

$$
\begin{aligned}
\delta E_{\mathrm{ph}}=-\left(2 m_{\mathrm{d}} \Delta\right)^{3 / 2} \frac{1}{V_{\mathrm{BZ}}} \times & \\
& \times \int_{S_{\mathrm{F}}} \mathrm{d} \sigma\left(\mathbf{K}_{\mathrm{F}}\right)\left\langle\frac{A(q)}{\hbar \omega_{q}}\right\rangle_{\text {pol }} 3 k_{\mathrm{B}} T
\end{aligned}
$$


où ici encore, $\delta E_{\mathrm{ph}}$ est négatif. Répétons que (14) n'est valide que pour $\Delta>0$.

En conclusion, $E_{\mathrm{ph}}$ présente une anomalie en $\left(V-V_{\mathrm{c}}\right)^{3 / 2}$ (on suppose la dérivée en $V$ de $\Delta, \Delta_{\mathrm{c}}^{\prime}$, strictement positive) dont le coefficient a un signe bien défini (négatif) et peut être calculé connaissant la matrice diélectrique. La pression $-\left[\partial E_{\mathrm{ph}} / \partial V\right]_{T}$ comporte une contribution positive anormale $\delta P_{\mathrm{ph}}$ en $\left(V-V_{\mathrm{c}}\right)^{3 / 2}$ pour $V>V_{\mathrm{c}}$. La pression totale est donc de la forme (posant $\left.x=V / V_{c}-1\right)$

$$
P=P\left(V_{\mathrm{c}}\right)-b x+c x^{1 / 2}+0\left(x^{3 / 2}\right)
$$

où $b$ et $c$ sont positifs. Le module de compressibilité $-V \partial P / \partial V$ vaut

$$
B(V)=-\frac{\partial P}{\partial x}=b-\frac{1}{2} c x^{-1 / 2}+0\left(x^{1 / 2}\right) .
$$

Ainsi $B$ s'annule et change de signe pour une valeur positive de $x\left(B\left(V_{c}^{+}\right)=-\infty\right)$. Le métal devient donc instable vis-à-vis d'une contraction du volume, à partir d'un volume strictement supérieur à $V_{\mathrm{c}}$.

$\mathrm{Ce}$ résultat repose sur les approximations énoncées dans l'introduction. Nous discuterons seulement très brièvement celle qui consiste à négliger l'effet de l'agitation thermique sur $\chi$. L'anomalie en $\Delta^{3 / 2}$ repose sur l'existence d'une surface de Fermi précise et ne peut donc exister qu'à $T=0 \mathrm{~K}$. Cela suffit néanmoins pour prouver l'instabilité du réseau pour des températures finies $T \gtrless T^{*}$, à l'aide de l'argument de continuité suivant. $B(V)$ s'annule à $0^{\circ}$ pour
$V=\tilde{V}_{0}>V_{\mathrm{c}}$. Lorsque $T$ croît, le volume $\tilde{V}_{\mathrm{T}}$ où $B$ change de signe se déplace (probablement vers $V_{c}$ ) et n'atteint $V_{\mathrm{c}}$ que pour une valeur finie $T^{*}$ de $T$. On ne peut rien dire $a$ priori quant à la valeur précise de $T^{*}\left({ }^{1}\right)$, et un effort supplémentaire sera nécessaire pour décider si cette théorie s'applique, par exemple, à la transition isostructurale CsII $\rightarrow$ CsIII évoquée dans l'introduction.

A cette anomalie en $-\Delta^{3 / 2}$ (pour l'énergie libre) vient s'ajouter l'anomalie purement électronique en $-\Delta^{5 / 2}$ associée à la formation d'une poche lorsque $\Delta<0$ [1]. Comme l'a montré Lifshitz [1], cette anomalie peut à elle seule induire une transition de phase isostructurale (N. F. Mott - non publié a fait un raisonnement analogue pour prédire une transition du premier ordre lors du passage semiconducteur-semi-métal par croisement de bande). En effet, le module de compressibilité correspondant s'écrit, pour $x=V / V_{c}-1<0$,

$$
B=b-\frac{3}{2} C|x|^{1 / 2}+0(x)
$$

et peut s'annuler pour $x$ raisonnablement petit si $C / b$ est suffisamment grand. Comparant (17) à (16), on constate que l'anomalie de $B$, d'origine purement électronique, est plus faible (en $-|x|^{1 / 2}$ ) que celle d'origine vibratoire (en $-x^{-1 / 2}$ ) qui induit nécessairement une transition de phase, de sorte que la conclusion énoncée plus haut reste inchangée.

( $\left.{ }^{1}\right)$ Sauf que $k_{\mathrm{B}} T^{*}$ doit être en ordre de grandeur voisin de $\Delta\left(\tilde{V}_{0}\right)$, valeur du gap pour lequel $B=0$ (remarque du rapporteur).

\section{Bibliographie}

[1] LifshitZ, I. M., Zh. Eksp. \& Teor. Fiz. 38 (1960) 1569 (Sov. Phys. JETP 11 (1960) 1130).

[2] Barisic, S., Labbe, J. et Cyrot-Lackman, F., J. Physique 30 (1969) 955.

[3] Kohn, W., Phys. Rev. 126 (1962) 1693.

[4] Hall, H. T., Merril, L. et Barnetr, J. D., Science 146 (1964) 1297.

[5] Yamashita, J. et Asano, S., J. Phys. Soc. Japan 29 (1970) 264.

[6] Louis, S. G. et Cohen, M. L., Phvs. Rev. 10 (1974) 3237.
[7] Ratti, V. K. et Jain, A., J. Phys. F. Metal Phys. 3 (1973) L 69. [8] McWhan, D. B., Parisot, G. et Bloch, D. B., J. Phys. F. Metal Phys. 4 (1974) L 69.

[9] Pick, R., Cohen, M. H. et Martin, R. M., Phys. Rev. B 1 (1970) 910.

[10] Maradudin, A. A., Montroll, E. W., Weiss, G. H. et IpaTOVA, I. P., Theory of Lattice Dynamics in the Harmonic Approximation (Academic Press, New York and London) 1971. 